\title{
Interferências em exames laboratoriais: critério diagnóstico para o diabetes mellitus e principais fármacos hipoglicemiantes
}

\author{
Interferences in laboratory tests: diagnostic criteria \\ for diabetes mellitus and major hypoglycemic agents
}

Daniela Hisaye KANASHIRO ${ }^{1}$, Flávia Domingues GAMEIRO $^{1}$, Válter Luiz da COSTA JÚNIOR ${ }^{1}$, Alexsandro Macedo SILVA ${ }^{1}$, Luciane Maria RIBEIRO NETO*1,2 Reiko Soraya MATSUI ${ }^{3}$, Sandro Jorge JANUÁRIO ${ }^{4}$, Paulo Caleb Júnior de Lima SANTOS ${ }^{1}$ Centro Universitário São Camilo, São Paulo, SP, Brasil. ${ }^{2}$ Departamento de Medicina da UNIFESP, São Paulo, SP, Brasil. ${ }^{3}$ Gerência técnica do Centro de Diagnóstico Mello, São Paulo, SP, Brasil. ${ }^{4}$ Centro de Virologia-Núcleo de Doenças Sanguíneas e Sexuais, Instituto Adolfo Lutz de São Paulo, São Paulo, SP, Brasil. Laboratório de Genética e Cardiologia Molecular do Instituto do Coração da Faculdade de Medicina da Universidade de São Paulo, SP, Brasil. E-mail:lumrn@hotmail.com

\begin{abstract}
Diabetes mellitus (DM) is among the most significant chronic conditions of health. Current diagnostic criteria for DM suggest the following laboratory tests: fasting plasma glucose, glycated or glycosylated hemoglobin, oral glucose tolerance test, fructosamine, insulin, C-peptide, glucagon. This laboratory tests may present drug interferences, which are physiological or analytical. It was possible identify 30 drugs or group of drugs that have evidences of interfering in the major diagnostic tests of DM according reviewed literature. It is important to note that virtually in its entirety these drugs interfere in the blood glucose levels, and $81.5 \%$ of these contribute to increase these values. These drugs include OTCs (over-the-counter drugs), as paracetamol and acetylsalicylic acid, and steroidal anti-inflammatory, as dexamethasone and prednisolone, extensively used in the drug therapy. Likewise, drugs used for treatment of diabetic patients included in the RENAME as NPH insulin (neutral protamine Hagedorn), regular human insulin, glibenclamide, glicazide and metformin hydrochloride may interfere in the laboratory tests. Early diagnosis and appropriate treatment are needed in order to prevent major damage for patients presenting this under-diagnosed complex disease. In this context, drug interferences are significant findings in the laboratory tests that evaluate DM and should be acknowledged by involved healthcare professionals.
\end{abstract}

KEYWORDS: Diabetes mellitus; Drugs Adverse Reaction; Diagnostic Techniques and Procedures.

\section{RESUMO}

O diabetes mellitus (DM) está entre as principais condições crônicas de saúde. O critério diagnóstico atual para o DM propõe os seguintes testes laboratoriais: glicose plasmática em jejum, hemoglobina glicada ou glicosilada, teste oral de tolerância à glicose, frutosamina, insulina, peptídeo $\mathrm{C}$ e glucagon. Estes exames laboratoriais podem apresentar interferências de medicamentos, sejam fisiológicas ou analíticas. Na literatura consultada foi possível identificar 30 fármacos ou grupo de fármacos que possuem evidências de interferir nos principais exames de diagnóstico da DM. Ressalta-se que praticamente na sua totalidade estes interferem nos níveis de glicemia, sendo que, 81,5\% destes contribuem de forma a aumentar estes valores. Dentre estes fármacos encontram-se MIPs (medicamentos isentos de prescrição), como o paracetamol e o ácido acetilsalicílico, e anti-inflamatórios esteroidais, como a dexametasona e prednisolona, amplamente utilizados na terapêutica medicamentosa. Da mesma forma, os medicamentos empregados no tratamento de pacientes diabéticos incluído na RENAME como insulina NPH (protamina neutra Hagedorn), insulina humana regular, glibenclamida, gliclazida e cloridrato de metformina podem interferir em exames laboratoriais. $\mathrm{O}$ diagnóstico precoce e o tratamento adequado se fazem necessários a fim de prevenirem maiores danos aos portadores desta doença complexa sub diagnosticada. Neste contexto, as interferências medicamentosas são importantes achados nos exames laboratoriais que avaliam o DM e devem ser reconhecidas pelos profissionais de saúde envolvidos.

PALAVRAS-CHAVE: Diabetes mellitus; Reação Adversa a Medicamentos; Técnicas e Procedimentos Diagnósticos 


\section{LISTA DE ABREVIAÇÕES:}

$\begin{array}{ll}\text { ADA } & \text { American Diabetes Association } \\ \text { ALT } & \text { alanina aminotransferase } \\ \text { AST } & \text { aspartato aminotransferase } \\ \text { DM } & \text { Diabetes mellitus } \\ \text { HbA1C } & \text { hemoglobina glicada ou glicosilada } \\ \text { HDL } & \text { lipoproteínas de alta densidade } \\ \text { LDL } & \text { lipoproteína de densidade baixa } \\ \text { NPH } & \text { protamina neutra Hagedorn }\end{array}$

RENAME Relação Nacional de Medicamentos Essenciais VLDL lipoproteína de densidade muito baixa

\section{INTRODUÇÃO}

O diabetes mellitus (DM) está entre as principais condições crônicas de saúde segundo o Sistema de Cadastramento e Acompanhamento de Hipertensos e Diabéticos. No Brasil, no ano de 2010, segundo o Ministério da Saúde, o número de diabéticos era de 40556 indivíduos, sendo 11078 do Tipo 1 e 29478 do Tipo 2. Observa-se também maior prevalência no gênero feminino, representando 56,4\% dos casos. No estado de São Paulo registrou-se 7029 casos (17,3\%) (1). Um estudo multicêntrico estimou a prevalência do DM em 7,6\%. Porém, sabe-se que estes números são subestimados devido ao grande número de diabéticos não diagnosticados (2).

Esta doença metabólica apresenta alta taxa de morbidade e de mortalidade por ser uma das principais causas de cegueira, de amputação de membros inferiores, de insuficiência renal e de doenças cardiovasculares, com impacto significativo na qualidade de vida das pessoas acometidas, o que requer cuidados clínicos e uma educação contínua aos profissionais de saúde e aos pacientes. Esta enfermidade eleva o custo médico-social, principalmente pelas suas complicações, e apesar de avanços científicos e tecnológicos, tem-se registrado aumento na sua incidência no Brasil e no mundo (3-6).

O DM apresenta graus variáveis de deficiência e ausência da insulina e/ou resistência insulínica e pode ser classificado em (7):

DM tipo 1: causada basicamente pela destruição seletiva das células pancreáticas e por deficiência grave ou absoluta de insulina, sendo a administração de insulina essencial para os indivíduos portadores deste tipo de DM. A destruição das células pode ser causada por mecanismo autoimune (mais comum) ou idiopática. É diagnosticada em maior frequência em pessoas com menos de 30 anos, porém podem manifestar-se nas demais faixas etárias.

DM tipo 2: caracteriza-se pela resistência tecidual à ação da insulina, em conjunto ou não com a deficiência relativa na secreção deste hormônio. Normalmente está associada à obesidade e ao envelhecimento, ocorrendo principalmente em pessoas com mais de 30 anos. O comprometimento na ação da insulina também afeta o metabolismo dos lipídios, resultando em aumento no fluxo de ácidos graxos livres e nos níveis de triglicerídeos e, de maneira recíproca, em baixos níveis de lipoproteínas de alta densidade (HDL). Os pacientes com DM tipo 2 podem ou não necessitar de insulina no tratamento hipoglicêmico.

Outros tipos específicos de diabetes: envolvem os defeitos genéticos das células $\beta$ pancreáticas, os defeitos genéticos na ação da insulina, as doenças do pâncreas exócrino, as endocrinopatias, o diabetes quimicamente induzido ou induzido por drogas, as formas incomuns de diabetes imuno-mediado e outras síndromes genéticas.

Diabetes gestacional: é definido como qualquer grau de intolerância à glicose com início ou primeira detecção durante a gravidez.

As causas das alterações no metabolismo dos carboidratos estão possivelmente relacionadas à dieta inadequada, à falta de exercícios físicos, à diminuição da massa magra corporal e, consequentemente à secreção alterada e à resistência insulínica. Os tratamentos do DM e das complicações geradas representam maior custo do que o investimento em ações de promoção à saúde e de prevenção. Além dos gastos financeiros com seguros, benefícios, internações e medicamentos, há o custo social observado na incapacidade física, absenteísmo e aposentadoria precoce $(5,8)$.

\section{CRITÉRIO DIAGNÓSTICO ATUAL PARA O DIABETES MELLITUS E AS POSSÍVEIS INTERFERÊNCIAS DE MEDICAMENTOS}

O critério diagnóstico atual para o DM, segundo a American Diabetes Association (ADA) e a Sociedade Brasileira de Diabetes (9), propõem os seguintes testes laboratoriais e respectivos valores:

Glicose plasmática em jejum: realizada na ausência de ingestão calórica por no mínimo 8 horas e valores $\geq 126 \mathrm{mg} / \mathrm{dL}$ indicam o DM. Além disso, há a indicação de DM para valores $\geq 200 \mathrm{mg} / \mathrm{dL}$, para glicemia realizada aleatoriamente, associados com sintomas clássicos de hiperglicemia ou crise hiperglicêmica.

Hemoglobina glicada ou glicosilada (HbA1C): bastante utilizada para avaliar o tratamento hipoglicêmico, pois sua medida avalia o período aproximado de 90 dias. Segundo a ADA, este exame laboratorial é também critério diagnóstico, sendo valores $\geq 6,5 \%$ indicativos do DM. Em pacientes portadores de hemoglobinopatias, a determinação da hemoglobina glicada não é recomendada, deste modo, a frutosamina pode ser realizada para o monitoramento do tratamento farmacológico. 
Teste oral de tolerância à glicose: indicado principalmente nos casos de valores de glicemia entre $>100 \mathrm{mg} / \mathrm{dL}$ e $<126 \mathrm{mg} / \mathrm{dL}$ ou, em suspeita de diabetes gestacional. Valores $\geq 200 \mathrm{mg} / \mathrm{dL}$ para glicose, após a ingestão da sobrecarga de 75 gramas de glicose anidro dissolvidas em água, são indicativos de DM.

Além dos exames laboratoriais citados acima, os exames de frutosamina, insulina, peptídeo $\mathrm{C}$ e glucagon podem ser realizados com as finalidades de monitoramento ou de auxiliar no diagnóstico do DM.

Frutosamina: os níveis no sangue refletem o controle da glicemia nas últimas duas a três semanas. O exame, contudo, não deve ser usado para diagnóstico de DM. Pode ser útil para a avaliação de alterações do controle do DM em intervalos menores, para julgar a eficácia de mudança terapêutica e para seguimento de gestantes com DM. A ADA recomenda a dosagem de frutosamina quando a $\mathrm{HbA1C}$ não é considerada um bom parâmetro de seguimento, como nos seguintes casos: rápidas alterações no tratamento do DM, DM gestacional, em pacientes com hemoglobinopatias e anemias diversas.

Insulina: vários mecanismos de resistência insulínica estão sendo descritos e a forma mais comum é a que acompanha a síndrome metabólica gerada pela obesidade. Neste caso, a dosagem de insulina é útil para a estimativa da resistência insulínica e esta geralmente apresenta valores elevados acompanhada de valores normais ou aumentados para a glicemia. Também é utilizada no diagnóstico de insulinoma.

Peptídeo C: auxilia no diagnóstico diferencial de hipoglicemia, na classificação de $\mathrm{DM}$, na verificação da função das células $\beta$ pancreáticas e na avaliação de transplante de pâncreas. A meia-vida do peptídeo C no plasma é cerca de 5 vezes maior que a meia-vida da insulina, por isso o peptídeo C é um indicador mais estável da secreção de insulina.

Glucagon: auxilia no diagnóstico de glucagonoma - tumores que em geral se originam no pâncreas, secretam quantidades excessivas de glucagon e podem causar a síndrome do glucagonoma. Existem três diferentes síndromes. A primeira consiste em um característico prurido de pele, um eritema necrolítico migratório, DM ou tolerância alterada à glicose, perda de peso, anemia e trombose venosa. Esta forma mostra geralmente níveis muito elevados do glucagon. A segunda forma está associada com o DM severa, e a terceira forma com síndrome múltipla da neoplasia da glândula endócrina. Esta forma pode ter níveis relativamente mais baixos do glucagon.

Os exames laboratoriais utilizados na avaliação do DM podem apresentar interferências de medicamentos, quer sejam fisiológicas ou analíticas. A Tabela 1 apresenta alguns exames e os principais medicamentos que podem gerar as interferências.

\section{MEDICAMENTOS HIPOGLICEMIANTES E AS POSSÍVEIS INTERFERÊNCIAS EM EXAMES LABORATORIAIS}

O tratamento do paciente diabético tem como objetivo a redução de complicações inerentes à doença, para isso são necessárias medidas não farmacológicas e/ou farmacológicas. Para a primeira, exige-se a mudança de estilo de vida, que engloba a perda gradual de peso, a realização de exercícios físicos e a alimentação saudável (6).

Quanto aos tratamentos farmacológicos, estes se baseiam: na reposição da insulina, na estimulação de sua secreção pelo pâncreas realizado por fármacos secretagogos, na diminuição da resistência à insulina ou na menor absorção de carboidratos.

De acordo com a Relação Nacional de Medicamentos Essenciais - RENAME (14), os medicamentos listados para o tratamento do DM para a população brasileira são as insulinas e os hipoglicemiantes orais: insulina NPH (protamina neutra Hagedorn), insulina humana regular, glibenclamida, gliclazida e cloridrato de metformina. A Tabela 1 apresenta as interferências mais comumente observadas.

\section{Insulinas}

A insulina promove o armazenamento de gordura e de glicose no interior das células-alvo especializadas e influencia o crescimento celular e as funções metabólicas de uma ampla variedade de tecidos. O objetivo da insulinoterapia consiste em repor a insulina basal normal (noturna, em jejum e entre as refeições) e a insulina prandial (durante as refeições). Esta terapia é utilizada há mais de 70 anos; no entanto, somente em 1982 foi produzida a insulina humana derivada de ácido desoxirribonucleico recombinante, tomando o lugar das insulinas de origem suína ou bovina. Além disso, desenvolveram-se os análogos da insulina, os quais modulam a atividade e a intensidade de ação da insulina (15-17).

Pacientes com DM tipo 1 são tratados com insulina, além destes, uma frequência considerável de pacientes com DM tipo 2 podem vir a necessitar da suplementação com o hormônio.

Insulina NPH e insulina humana regular (interferências em exames laboratoriais): as concentrações séricas de fosfato inorgânico, magnésio e potássio podem diminuir (12). 
Tabela 1 - Principais exames laboratoriais utilizados na avaliação do diabetes mellitus e os principais medicamentos que podem gerar interferências (10-13).

\begin{tabular}{|c|c|c|}
\hline EXAME & MEDICAMENTO & INTERFERÊNCIA \\
\hline \multirow{27}{*}{ Glicemia } & ácido acetilsalicílico & Diminui \\
\hline & ácido ascórbico & Diminui \\
\hline & amitriptilina & Aumenta/ Diminui \\
\hline & baclofeno & Aumenta \\
\hline & carbamazepina & Aumenta \\
\hline & cafeína & Aumenta \\
\hline & dexametasona & Aumenta \\
\hline & domperidona & Diminui \\
\hline & estrogênios & Aumenta \\
\hline & estradiol & Aumenta \\
\hline & fenitoína & Aumenta \\
\hline & haloperidol & Aumenta \\
\hline & hidroclorotiazida & Aumenta \\
\hline & imipramina & Aumenta \\
\hline & iodeto de pralidoxime & Aumenta \\
\hline & isoniazida & Aumenta \\
\hline & levodopa & Aumenta \\
\hline & levotiroxina & Aumenta \\
\hline & lítio & Aumenta \\
\hline & meperidina & Aumenta \\
\hline & moxifloxacino & Diminui \\
\hline & nortriptilina & Aumenta \\
\hline & paracetamol & Diminui \\
\hline & piperacilina + tazobactam & Aumenta \\
\hline & prednisolona & Aumenta \\
\hline & sidenafila & Aumenta \\
\hline & teofilina & Aumenta \\
\hline \multirow{3}{*}{ Hemoglobina glicada } & álcool (etanol) & Aumenta \\
\hline & hidroclorotiazida & Aumenta \\
\hline & opióides & Aumenta \\
\hline Insulina & enalapril & Diminui \\
\hline Glucagon & hidroclorotiazida & Diminui \\
\hline
\end{tabular}




\section{Sulfonilureias}

As sulfonilureias são úteis nos estágios iniciais do DM tipo 2. Surgiram casualmente, após observar-se que um derivado da sulfonamida (utilizado no tratamento de febre tifóide) causou hipoglicemia.

Seu mecanismo de ação se dá por estimulação da secreção de insulina, através da redução dos níveis séricos de glucagon e fechamento dos canais de potássio nos tecidos extra-pancreáticos, reduzindo assim a concentração plasmática da glicose $(12,18)$.

Segundo a RENAME (14), as sulfonilureias disponíveis na rede pública de saúde são: glibenclamida 5 mg (comprimido) e gliclazida $30 \mathrm{mg}$ (comprimido de liberação controlada) e $80 \mathrm{mg}$ (comprimido de uso restrito a idosos).

Glibenclamida (interferências em exames laboratoriais): há relatos de casos de distúrbios hematopoiéticos, como por exemplos, trombocitopenia, anemia e leucopenia, que podem progredir para agranulocitose.

Houve relato de alguns casos de anemia hemolítica e vasculite. Em geral, tais alterações são reversíveis após a interrupção do uso do medicamento. Raramente podem ocorrer alterações hepáticas, como: elevação dos níveis de enzimas hepáticas, hepatite e colestase, distúrbios mais graves das funções hepáticas e alteração do sistema endócrino, com prejuízo do controle metabólico da hiperglicemia.

Gliclazida (interferências em exames laboratoriais): alterações hematológicas são raras e incluem anemia, leucopenia, trombocitopenia e granulocitopenia. As alterações geralmente são reversíveis quando se interrompe o tratamento.

Alterações hepatobiliares: aumento nos níveis das enzimas hepáticas (aspartato aminotransferase - AST, alanina aminotransferase - ALT, fosfatase alcalina) e hepatite em casos isolados.

\section{Biguanidas}

Sua ação não depende da presença de células funcionais do pâncreas, porém seu mecanismo de ação ainda não está totalmente elucidado. Sua ação pode ocorrer por captação direta da glicólise, a qual é utilizada na musculatura esquelética, diminuição da gliconeogênese hepática e renal, redução do nível de glicemia, de LDL e de VLDL (lipoproteínas de densidades baixa e muito baixa), redução da absorção de glicose pelo trato gastrintestinal, aumento da conversão da glicose em lactato pelos enterócitos e a diminuição dos níveis plasmáticos de glucagon $(16,18)$.

Segundo a RENAME (14), a biguanida disponível na rede pública de saúde é o cloridrato de metformina 500 e $850 \mathrm{mg}$ (comprimidos).

Cloridrato de metformina (interferências em exames laboratoriais): pode causar resultado falso positivo em teste de cetona urinária, além de diminuir as concentrações séricas do colesterol total, do LDL e dos triglicerídeos. O lactato plasmático e o HDL podem estar aumentados (12).

\section{CONCLUSÃO}

O diagnóstico precoce e o tratamento adequado se fazem necessários a fim de prevenir maiores danos aos portadores desta doença complexa sub-diagnosticada. Neste contexto, as interferências medicamentosas são importantes achados nos exames laboratoriais que avaliam o DM e devem ser reconhecidas pelos profissionais de saúde envolvidos.

\section{REFERÊNCIAS}

1. BRASIL. Ministério da Saúde. DATASUS. 2011 [cited 2013 05/15]: Available from: http://hiperdia.datasus.gov.br/hiperelhiperrisco.asp.

2. Malerbi DA, Franco LJ. The Brazilian cooperative group on the study of diabetes prevalence. multicenter study of the prevalence of diabetes mellitus and impaired glucose tolerance in the urban Brazilian population aged 30-69 yr. Diabetes Care. 1992; 15(11): 1509-16.

3. Neri AL. Idosos no Brasil: vivências, desafios e expectativas na terceira idade. 1 ed. São Paulo: Fundação Perseu Abramo, 2007.

4. Cryer PE. Hypoglycemia in type 1 diabetes mellitus. Endocrinol Metabolism. Clinics of North America. 2010; 39(3): 641-54.
5. McCarthy MI. Genomics, type 2 diabetes, and obesity. New England J Med. 2010; 363(24): 2339-50.

6. Cryer PE. Death during intensive glycemic therapy of diabetes: mechanisms and implications. Am J Medicine. 2011; 124(11): 993-6.

7. Report of the expert committee on the diagnosis and classification of diabetes mellitus. Diabetes Care. 2003; 26(S1): 5-20.

8. Tavares DMS, Guidetti GECB, Moreira MIBS. Características sócio-demográficas, condições de saúde e utilização de serviços de saúde por idosos. Rev Eletr Enfermagem. [Internet]. 2008; 10(2):299-309. Available from: http://www.fen.ufg.br/ revista/ v10/n2/v10n2a02.htm. 
9. Executive summary. Standards of medical care in diabetes 2011. Diabetes Care. 2011; 34(S1): 4-10.

10. Ceriello A, Giugliano D, Dello Russo P, Sgambato S, D'Onofrio F. Increased glycosylated hemoglobin A1 in opiate addicts. Evidence for hyperglycemic effect of morfine. Diabetologia. 1962; 22(5): 379.

11. Goldstein DE, Wiedmeyer HM, England JD, Little RR, Parker KM. Recent advances in glycosylated hemoglobin measurements. Critical Rev Clinical Lab Sc. 1984; 21(3): 187-228.

12. Zanini AC, Basile AC, Follador W, Oga S. Guia de Medicamentos. 2 ed. São Roque: Ipex Editora. 1997.

13. Ferreira B, Santos K, Rudolph S, Alcanfor J, Cunha L. Estudo dos medicamentos utilizados pelos pacientes atendidos em laboratório de análises clínicas e suas interferências em testes laboratoriais: uma revisão da literatura. Rev Eletron Farmácia. 2009; 6(1): 33-43.
14. BRASIL. Relação nacional de medicamentos essenciais - Rename 2010. 7 ed. Brasília: Ministério da Saúde. 2010.

15. Craig CR, Stitzel RE. Farmacologia moderna: com aplicações clínicas. 6 ed. Rio de Janeiro: Guanabara Koogan. 2005.

16. Katzung BG. Farmacologia - Básica \& Clínica. 9 ed. Rio de Janeiro: Guanabara Koogan. 2005.

17. Cryer PE, Axelrod L, Grossman AB, Heller SR, Montori VM, Seaquist ER, Service FJ. Endocrine Society. Evaluation and management of adult hypoglycemic disorders: an Endocrine Society Clinical Practice Guideline. J Clin Endocrinol Metabolism. 2009; 94(3): 709-28.

18. Rang HP, Dale MM. Farmacologia. 6 ed. Rio de Janeiro: Elsevier. 2007. 